\title{
Radiation doses in CT examinations from the West China Hospital, Sichuan University and setting local diagnostic references levels
}

\author{
Zhenlin $\mathrm{Li}^{1}{ }^{\wedge}$, Jinge Zhang ${ }^{1} \wedge$, Chunchao $\mathrm{Xia}^{1}$, Fei Zhao ${ }^{1}$, Kai Zhang ${ }^{1}$, Yuming $\mathrm{Li}^{1}$, Lei $\mathrm{Li}^{1}$, Jin $\mathrm{Pu}^{1}$, \\ Wanlin Peng ${ }^{1}$, Keling Liu ${ }^{1}$, Yingkun Guo ${ }^{2}$ \\ ${ }^{1}$ Department of Radiology, West China Hospital, Sichuan University, Chengdu, China; ${ }^{2}$ Department of Radiology, West China 2nd University \\ Hospital, Sichuan University, Chengdu, China \\ Contributions: (I) Conception and design: Y Guo, Z Li; (II) Administrative support: None; (III) Provision of study materials or patients: None; (IV) \\ Collection and assembly of data: C Xia, F Zhao, K Zhang; (V) Data analysis and interpretation: Y Li, L Li, J Pu, W Peng, K Liu; (VI) Manuscript \\ writing: All authors; (VII) Final approval of manuscript: All authors. \\ Correspondence to: Yingkun Guo. Department of Radiology, West China 2nd University Hospital, Sichuan University, No. 20 Third Section of \\ Renmin South Road, Chengdu, China. Email: gykpanda@163.com.
}

\begin{abstract}
Background: Our study aims to summarize the data of radiation doses collected from consecutive CT examinations by using the Radiometrics software and contributing to the establishment of the region's diagnostic reference levels (DRLs).

Methods: The radiation doses in 158,463 CT examinations performed on 106,275 adults between April 2017 and April 2019 were retrospectively analyzed. The median value and interquartile range (IQR) of volumetric CT dose index (CTDIvol), dose-length product (DLP), effective dose (ED), and size-specific dose estimate (SSDE) were calculated according to the scanning region.

Results: The median CTDIvol (mGy) for each scanning region was 42.3 (head), 6.2 (chest), and 9.0 (abdomen). The median DLPs (mGy.cm) for single-phase, multi-phase, and all examinations were as follows: 607, 794, and 641 for the head; 220, 393, and 237 for the chest; 298, 1,141, and 570 for the abdomen. The median EDs (mSv) for single-phase, multi-phase, and all examinations are as follows: 1.6, 2.6, and 1.8 for the head; 5.1, 8.1, and 5.3 for the chest; 5.8, 20.3, and 10.4 for the abdomen.

Conclusions: Our study's results could provide a basis for the evaluation of CT scanning radiation dosage and supply evidence for the establishment of local DRLs in China's Sichuan Province.
\end{abstract}

Keywords: Radiation dosage; multidetector computed tomography; reference standards; quality control

Submitted Jun 29, 2020. Accepted for publication Aug 12, 2020.

doi: $10.21037 /$ atm-20-5443

View this article at: http://dx.doi.org/10.21037/atm-20-5443

\section{Introduction}

The development of multislice spiral computed tomography (MSCT) has led to a noticeable quantum leap in the clinical performance of CT, enabling a faster and correct diagnosis of diseases. As imaging techniques develop so rapidly, using CT has increased greatly in the past few years (1). Many new techniques including CT angiography (CTA), CT perfusion imaging (CTP), and gemstone spectral imaging
(GSI) are providing more and more valuable information for clinical diagnosis (2-5). However, the diagnostic benefits of CT imaging are associated with unavoidable radiation exposure. Exposure to the ionizing radiation of CT scans, even at relatively low doses, will bring incrementally increased risks of cancer, especially in women and young people (6-8). Although techniques of lowdose CT scanning are widely used in clinical applications

^ ORCID: Zhenlin Li, 0000-0001-7525-330X; Jinge Zhang, 0000-0001-5045-6530. 
nowadays, the radiation dose is still the biggest obstacle to popularizing using CT (3). With the public's growing recognition of the importance of radiation protection, dose management has become a research hot spot in the field of CT imaging (9-11). Nowadays, nearly all CT scanners are required to report radiation doses as some particular values, including volumetric CT dose index $\left(\mathrm{CTDI}_{\mathrm{vol}}\right)$ and dose-length product (DLP) (12). These values are usually given in a dose report by CT modality automatically and effective dose (ED) could be calculated from the organ dosage and tissue-weighting factors. However, a single dose report only reflects the dose level of a specific single examination. Diagnostic reference levels (DRLs) with big data of consecutive CT examinations could better reflect the average dose level in individual hospitals or regions and guide radiology facilities in quality improvement efforts to optimize CT workflow (13). Some researchers or health oversight organizations in the United States and Europe have investigated the DRLs of local hospitals (13-15). Although the National Health Commission of China has issued the national standard of the radiation dose for adults in CT scan, there are few studies from dose data of a large sample of the radiation dose, and there is also a lack of research on classification by single phase scan and multiple phase scan. And in China, the ethnic and physical differences of the population in different regions lead to a large gap in the height and weight of local patients treated in hospitals in different regions (for example, people in north China are usually taller and stronger than those in the south), which will affect the parameter setting of CT scans and the final radiation doses. In addition, different regions in China have very different levels of economic development, leading to a large gap in the performance of CT equipment in public hospitals. Considering patients from different regions may have substantial differences in average body size, as well as different equipment and workflow, it is necessary to establish more local diagnostic reference levels (LDRLs) in China.

The purpose of our study is to summarize the data of radiation doses collected from consecutive CT examinations in West China Hospital and to develop the DRLs of this region. We present the following article in accordance with the MDAR reporting checklist (available at http://dx.doi. org/10.21037/atm-20-5443).

\section{Methods}

\section{Patients}

This retrospective study was conducted according to the ethical guidelines of the Declaration of Helsinki (as revised in 2013) and approved by the Ethics Committee of West China Hospital. Written informed consent was obtained from all patients or their next of kin before the CT examination. All patients were informed of the benefits and risks of CT scans, the possibility that their data might be used in medical research, the privacy policy, and other related information. We collected the dose data of adults for the head, chest, and abdomen CT scans obtained between April 2017 and April 2019 from 10 scanners in West China Hospital.

\section{Assessment and statistics of radiation doses}

The scanning data were transferred from CT scanners to a single server of picture archiving and communication system (PACS) and found by Radimetrics (Bayer Healthcare, Whippany, NJ, USA). This software tool can identify and track the dose data from the dose report files on PACS, including the parameters of CTDI ${ }_{\mathrm{vol}}$ and DLP. Also, Radiometrics can calculate the size-specific dose estimate (SSDE) by calculating patient diameter from the middle-level scan length. And Radimetrics can also calculate ED automatically by using radiation exposure parameters and phantom libraries. ED is calculated on the organs exposed by the applied radiation multiplied by tissueweighting factors. In radiometrics, the organ doses are first calculated using Monte Carlo probabilistic simulations that account for scattered radiation using a library that includes standardized male and female anthropomorphic mathematical phantoms. The ED is estimated according to the published ICRP103 tissue-weighting factors $(16,17)$. The radiation dose was analyzed for single-phase and multiphase scans (including contrast-enhanced scans and angiography) separately. In multiphase scans, we averaged the SSDE and CTDIvol for each radiation event and added the DLPs and effective doses from each radiating event to obtain the total metric for the examination. And the brief scans obtained to determine the timing for the injection of iodinated contrast medium (monitoring trigger scans) were not included in the calculation.

According to the clinical requirements of our hospital, abdominal contrast-enhanced scans are generally performed in 3 phases (plain scan, late arterial phase, and vein phase), while other areas are generally performed in 2 phases. Abdominal CT angiography is generally performed in 4 phases (plain scan, arterial phase, portal vein phase, and vein phase), chest angiography is usually performed in 
Table 1 Details of the 10 CT scanners in this study

\begin{tabular}{lcccc}
\hline No. & Manufacturer of CT scanner & Model of CT scanner & Number of detector rows & Installation time \\
\hline CT 1 & Siemens & Somatom Definition AS & 64 & $2013-5-14$ \\
CT 2 & Philips & Brilliance 64 & 64 & $2006-11-29$ \\
CT 3 & Siemens & Somatom Definition AS & 64 & $2013-5-14$ \\
CT 4 & Siemens & Somatom Definition Flash & 64 & $2013-5-14$ \\
CT 5 & Siemens & Somatom Sensation 16 & 16 & $2003-11-1$ \\
CT 6 & Philips & Brilliance 16 & 16 & $2006-7-11$ \\
CT 7 & Siemens & Somatom Definition Flash & 64 & $2015-3-25$ \\
CT 8 & GE & Revolution & 256 & $2016-9-5$ \\
CT 9 & Siemens & Somatom Definition AS & 64 & $2012-12-7$ \\
CT 10 & Siemens & Somatom Definition AS & 64 & $2009-12-18$ \\
\hline
\end{tabular}

3 phases, and angiography of other examined areas is usually performed in 2 phases.

\section{CT modalities}

Scans were obtained from 10 CT scanners at three institutions of West China Hospital (including headquarter and two branches). Table 1 shows the specifications and other details of the ten scanners in this study.

\section{Statistical analysis}

These dose data were collected and analyzed on the Radimetrics platform so that we could get the median value and interquartile range (IQR) of CTDIvol, DLP, $\mathrm{ED}$, and SSDE for each examined region. We summarized dose data of all patients included, and compare the results with other similar studies. This study is a collection and summary of large sample data, without using statistical test methods.

\section{Results}

\section{Number of patients and examinations}

Totally 158,463 diagnostic CT examinations performed on 106,275 adults (men: $\mathrm{n}=52,463$; women: $\mathrm{n}=53,812$ ) were enrolled in our retrospective analysis, including 29,762 examinations for head $(18.8 \%$ of 158,463$), 73,591$ examinations for chest $(46.4 \%$ of 158,463$), 37,271$ examinations for abdomen $(23.5 \%$ of 158,463$)$, and 17,839 examinations for chest \& abdomen $(11.3 \%$ of 158,463$)$. The median age of all patients was $46.9 \pm 10.2$ years.

\section{Data of radiation doses}

The statistics of radiation dose parameters are reported in Table 2, including median value and IQR. In general, the 75 th percentiles are usually selected as DRLs. So, we used the 3rd quartile (Q3) as a simple point of reference from which hospitals of this area can compare their radiation doses. Table 3 summarizes the Q3 of dose data in our study.

\section{Comparison with other DRLs}

And we compared the DRLs (Q3) of CTDI ${ }_{\text {vol }}$ and DLP in our study with those in other researches or guidelines published in previous, including DRLs from the United States of America (13), UK (15), Japan (18), and China's Jilin (19) and Jiangsu (20) Province. The results are shown in Figures 1 and 2 [Zhou et al.'s (20) study did not provide DRLs of CTDI $\mathrm{vol}_{\mathrm{vol}}$. Therefore, data from China's Jiangsu province were unincluded in the comparison of Figure 1]. It is also worth mentioning that a singlephase scan (plain scan) and a multiphase scan (including contrast-enhanced CT scan and CT angiography) were studied separately in our study and the national standard of China. However, some research only included plain scan data or did not explicitly indicate it, so that the figures only included single-phase scan dose data of our study. 
Table 2 Radiation dose data of each examination type in our study (numbers in parentheses are SSDEs)

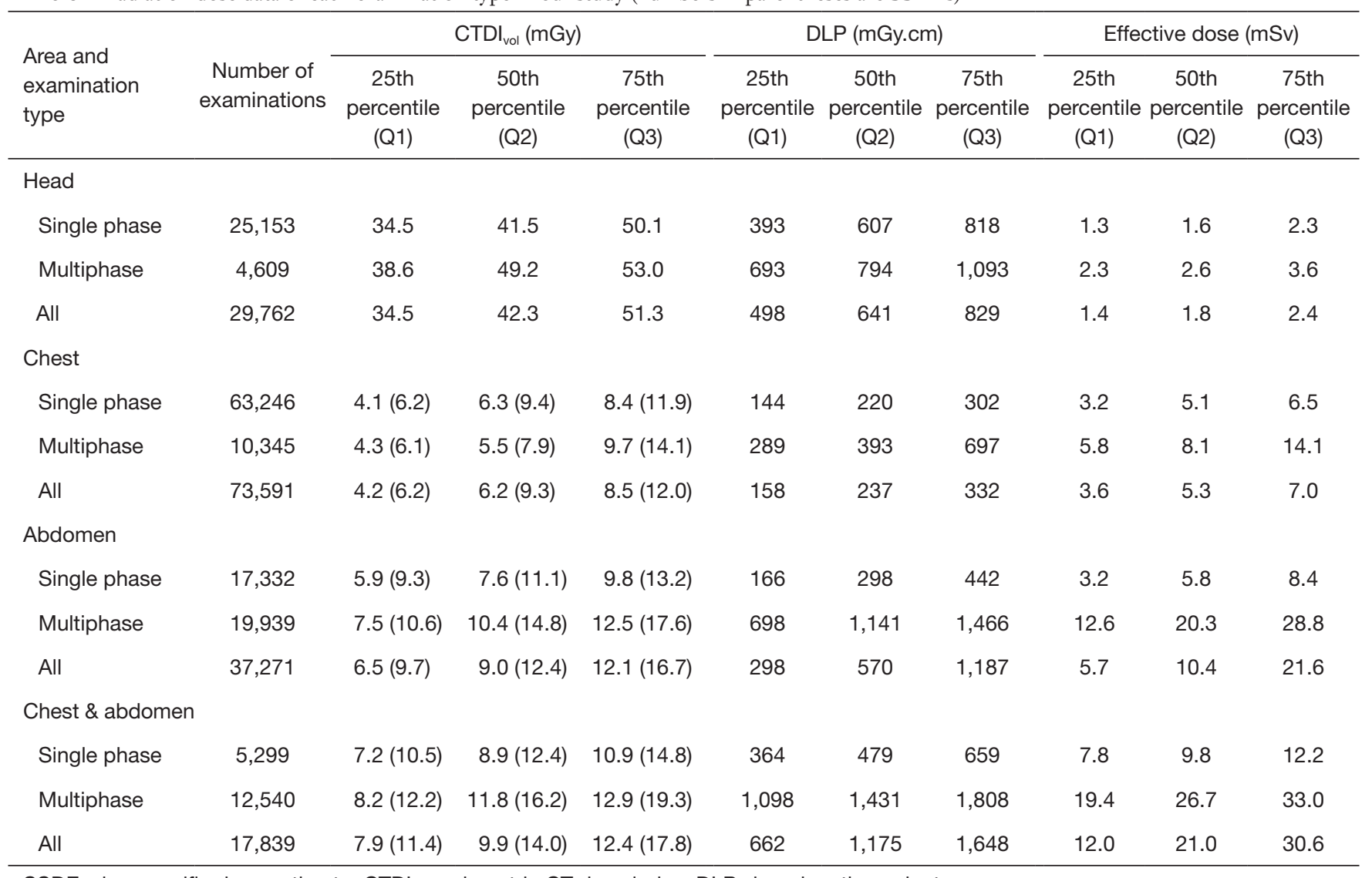

SSDE, size-specific dose estimate; CTDI $_{\text {vol }}$, volumetric CT dose index; DLP, dose-length product.

\section{Discussion}

Presently, radiation doses of CT scans are usually reported as two values: CTDI $_{\mathrm{vol}}$ and DLP (21), these two values are automatically calculated by CT scanners according to scanning parameters. $\mathrm{CTDI}_{\mathrm{vol}}$ is an index of average scanner output (mGy), which reflects specific scanning parameter choices in one scanning transverse slice related to radiation dose. DLP is the product of CTDI ${ }_{\mathrm{vol}}$ and scanning length (mGy $3 \mathrm{~cm})$. CTDI ${ }_{\mathrm{vol}}$ and DLP are determined for a $32 \mathrm{~cm}$ phantom (all body CT examinations: chest, abdomen, trunk, and spine) and a $16 \mathrm{~cm}$ phantom (head CT examination). However, neither CTDI $\mathrm{I}_{\mathrm{vol}}$ nor DLP indicates the specific factors of patients. The American Association of Physicists in Medicine (AAPM) task group 204 has introduced the concept of size-specific dose estimate (SSDE) to refine CTDI vol $_{\text {on }}$ patient size, derived from $\mathrm{CTDI}_{\mathrm{vol}}$, and measures patient diameters using lookup tables for conversion factors $(6,22,23)$. In other words, SSDE reflects an adjusted CTDI $_{\mathrm{vol}}$ measurement according to patient size. Calculating SSDE requires manual or automatic measurement of cross-sectional diameters of patients on transverse CT images or localizers, and requires complex calculation procedures, the main obstacle to the expansion of the practical application of $\operatorname{SSDE}(13,24)$. In our study, the software tool Radimetrics automatically calculated SSDEs. What is more, it also calculates effective dose (ED) by using the library of cristy phantoms and matching patients to a particular phantom on gender, age, weight, or diameter. SSDE and ED both consider the factors of patients so that they can reflect the radiation dose level more accurately. The Radiometric software obtained the DLP and CTDI ${ }_{\text {vol }}$ values by reading the dose report from the CT scanners, so these two values were accurate, while SSDE and ED were calculated, so their accuracy needs to be verified. Considering there have been many types of research directly using the results of Radimetrics, we did not analyze its accuracy $(13,19)$. The accuracy and scientificity of its calculation method could be explored in 
Table 3 Preliminary local CT DRLs from West China Hospital

\begin{tabular}{|c|c|c|c|}
\hline Area and examination type & $\mathrm{CTDI}_{\mathrm{vol}}$ (mGy) & DLP (mGy.cm) & Effective dose (mSv) \\
\hline Single phase & 50.1 & 818 & 2.3 \\
\hline Multiphase & 53.0 & 1,093 & 3.6 \\
\hline All & 51.3 & 829 & 2.4 \\
\hline Single phase & 8.4 & 302 & 6.5 \\
\hline Multiphase & 9.7 & 697 & 14.1 \\
\hline All & 8.5 & 332 & 7.0 \\
\hline \multicolumn{4}{|l|}{ Abdomen } \\
\hline All & 12.1 & 1,187 & 21.6 \\
\hline \multicolumn{4}{|l|}{ Chest \& abdomen } \\
\hline Single phase & 10.9 & 659 & 12.2 \\
\hline Multiphase & 12.9 & 1,808 & 33.0 \\
\hline All & 12.4 & 1,648 & 30.6 \\
\hline
\end{tabular}

DRL, diagnostic reference level; CTDI ${ }_{\text {vol }}$, volumetric CT dose index; DLP, dose-length product.

the follow-up study. The term diagnostic reference level (DRL) was first introduced in 1996 by the International Commission on Radiological Protection (ICRP) (25). The DRL process involves collecting dose data of consecutive examinations from participating institutions, usually set at the 75 th percentile of the histogram of aggregated dose data $(13,26)$. DRLs provide a means for practices comparing their radiation dose to benchmarks derived from aggregated dose data collected on a local, regional, or national level (25). By setting this dose reference level, it becomes possible to determine the facilities or units with higher doses for evaluation of the reason for such doses and perform optimization. The DRL process has been popularized in the United States, Europe, and Japan, and is applied with good results (13-15). In China, although the national standard of DRLs for adults in CT scans has been issued by the National Health Commission (27), there are still few studies on dose data of a large sample of patients or multicenter and cross-regional studies. Zhou et al. (20) and Zhou et al. (19) investigated radiation dose data from CT scans in Jiangsu and Jilin provinces of China to help establish their local DRLs, but these studies did not collect data of singlephase and multi-phase scans separately. Multiphase scans, including angiography and contrast-enhanced scans, often have higher radiation doses than plain scans because they require separate scans before and after contrast injections. Moreover, the exposure parameters of multi-phase scanning are different from those of plain scanning, so it is necessary to set up a reference standard for its separate classification statistics. It is also hoped it could be counted separately, or a more detailed classification of scanning scheme could be established in the future multi-center and cross-region research.

In our study, we collected dose data of consecutive clinical CT examinations over two years (Apr. 2017-Apr. 2019) at three institutions of West China Hospital (including headquarter and two branches, Yongning Branch and Shangjin Branch), and 158,463 examinations of 106,275 adults were enrolled. West China Hospital, namely West China Health Center, is the largest general hospital in Sichuan province of China, many CT examinations are performed in the department of radiology of West China Hospital daily. Meanwhile, more than 10 CT scanners from various manufactures (including Siemens, Philips, and GE) had been installed as of 2017 since 2003 (shown in Table 1), covering old and new imaging equipment. Therefore, the 


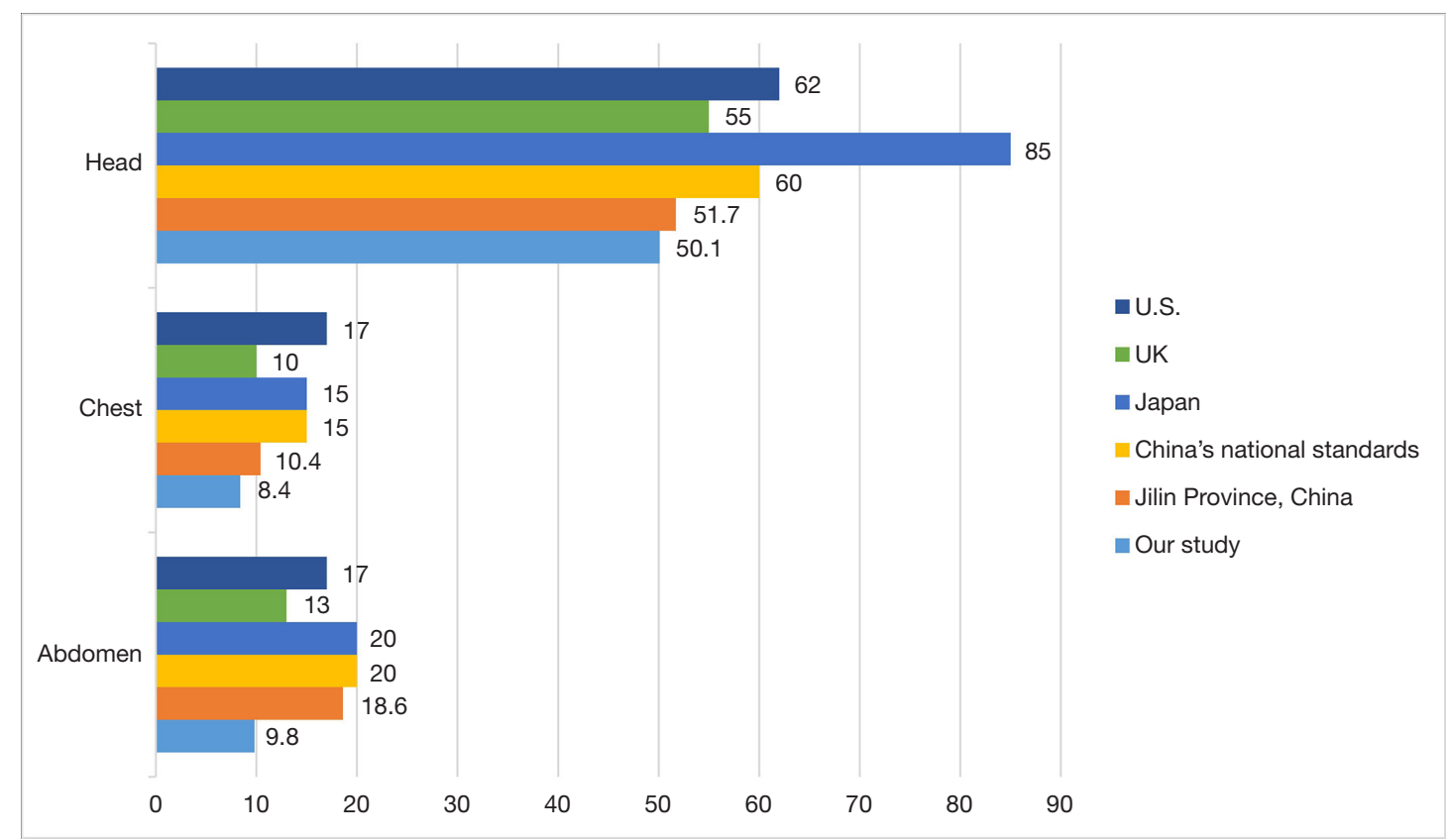

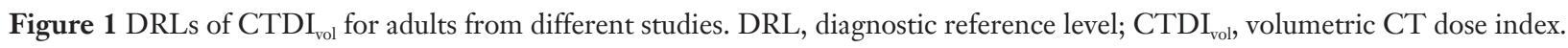

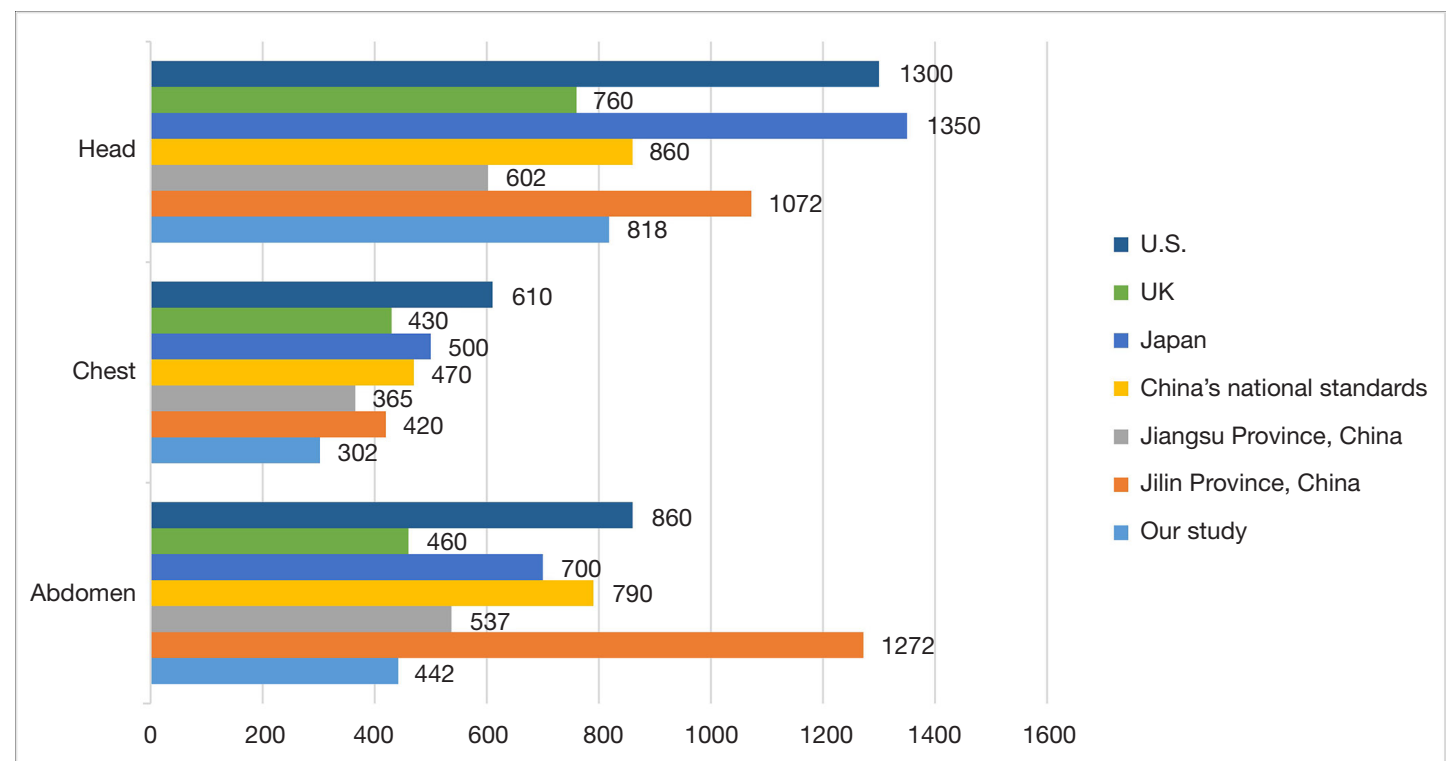

Figure 2 DRLs of DLP for adults from different studies. DRL, diagnostic reference level; DLP, dose-length product.

dose data we collected was representative to a certain extent and could help develop the DRLs of this hospital and this region. Institutions in Sichuan Province or other areas of China can use our metrics as a starting point to assess their CT dose levels.

We compared the local DRLs obtained in our study with
DRLs from guidelines or research in the United States, UK, and Japan. Our results showed similarly or lowered CTDIvol and DLP. Further, compared with China's national standards and the results of China's Jilin and Jiangsu provinces, our DRLs are also relatively lower (shown in Figures 1 and 2). These results may be attributed to the 
continuous progress of low-dose CT scanning technology, as well as the emphasis on radiation doses in West China Hospital and the corresponding measures taken. For dose optimization, we have several suggestions on the experience of West China Hospital. First, enable real-time automatic exposure control technology when scanning, including Siemens' Care Dose 4D (28), Care kV (29) technology, and so on. Secondly, the iterative reconstruction algorithm could significantly reduce the radiation dose on the premise of ensuring image quality, including Siemens' IRIS technology (30) and GE's ASIR and ASIR-V technology (31). Thirdly, the training and learning of CT technicians should be strengthened to construct CT scan workflow more standardized in the preparation of patients before the examination and the setting of scan parameters to accurately formulate the scanning plan and avoid rescanning due to operational errors. Fourthly, a complete radiation dose and image quality control system should be established to review and analyze the cases of excessive radiation doses regularly. As for the specific impact of these techniques or measures on the reduction of radiation dose, we are conducting further comparative research with the help of the Radimetrics software to explore it. It must be noted, due to the huge differences in patient ethnicity, patient body size, scanning sequence, scanning equipment, workflow and other factors between hospitals in each country or region, the differences between DRLs reported from different studies may not be as significant actually, but more about helping set up local DRLs. Moreover, the establishment of DRLs is mainly to provide reference for radiation dose control in local hospitals, but for a specific patient, the scanning parameters and radiation dose of CT scan should be optimized according to their body size, gender, age, medical history and other factors on the premise of meeting clinical diagnosis.

Our study has some limitations. First, only two years of data in one hospital were enrolled, meaning the sample size was small. Dose data from more hospitals and more patients need to be included, only then will DRLs on regional or national levels be set up. Most hospitals in the Sichuan province of China do not yet have Radiometrics, or similar software installed and not join the data-sharing network, we are not yet able to obtain more hospitals' data. It is expected the cloud platform of the Sichuan radiation quality control center under construction and the multi-center research will help a lot to set up the DRLs on the province level. Together, we hope West China Hospital and other hospitals will continue to update and add to these dose data for the creation of benchmarks, as only constantly updated DRLs are meaningful. Then, with some constraints, we only analyzed the examined area of the head, chest, and abdomen. More examined body parts should be included in a future study. And the categories are not detailed enough, for example, the examinations of the abdomen in our study included the upper abdomen, lower abdomen, whole abdomen, abdomen, angiography, and other types of abdomen CT scans. So, the value of our local DRLs for clinical guidance is limited, and further research is needed.

\section{Acknowledgments}

The authors acknowledge Sixian $\mathrm{Hu}, \mathrm{Xu} \mathrm{Xu}$, and Lingming Zeng for their significant help with data collection in this work.

Funding: This study has received funding by "1.3.5 project for disciplines of excellence, West China Hospital, Sichuan University" (Program Number: ZYGD18019), the recipient is $\mathrm{ZL}$.

\section{Footnote}

Reporting Checklist: The authors have completed the MDAR reporting checklist. Available at http://dx.doi.org/10.21037/ atm-20-5443

Data Sharing Statement: Available at http://dx.doi. org/10.21037/atm-20-5443

Conflicts of Interest: All authors have completed the ICMJE uniform disclosure form (available at http://dx.doi. org/10.21037/atm-20-5443). The authors have no conflicts of interest to declare.

Ethical Statement: The authors are accountable for all aspects of the work in ensuring that questions related to the accuracy or integrity of any part of the work are appropriately investigated and resolved. This retrospective study was conducted according to the ethical guidelines of the Declaration of Helsinki (as revised in 2013) and approved by the Ethics Committee of West China Hospital. Written informed consent was obtained from all patients or their next of kin before the CT examination.

Open Access Statement: This is an Open Access article distributed in accordance with the Creative Commons Attribution-NonCommercial-NoDerivs 4.0 International 


\section{Page 8 of 9}

License (CC BY-NC-ND 4.0), which permits the noncommercial replication and distribution of the article with the strict proviso that no changes or edits are made and the original work is properly cited (including links to both the formal publication through the relevant DOI and the license). See: https://creativecommons.org/licenses/by-nc-nd/4.0/.

\section{References}

1. Dreuil S, Etard C. Exposure of the French population to ionizing radiations from medical diagnostic procedures in 2012. Radioprotection 2017;52:45-9.

2. Shinohara Y, Sakamoto M, Kuya K, et al. Assessment of carotid plaque composition using fast-kV switching dual-energy CT with gemstone detector: comparison with extracorporeal and virtual histology-intravascular ultrasound. Neuroradiology 2015;57:889-95.

3. Li ZL, Li H, Zhang K, et al. Improvement of image quality and radiation dose of CT perfusion of the brain by means of low-tube voltage (70 KV). Eur Radiol 2014;24:1906-13.

4. Yang SM, Ko WC, Meng LH, et al. Single-stage hybrid localization: a combination of bronchoscopic lung mapping followed by post-mapping computed tomographic reconstruction and additional transthoracic needle localization in a cone beam computed tomography room. Ann Transl Med 2019;7:30.

5. Winklehner A, Karlo C, Puippe G, et al. Raw databased iterative reconstruction in body CTA: evaluation of radiation dose saving potential. Eur Radiol 2011;21:2521-6.

6. Khawaja RD, Singh S, Vettiyil B, et al. Simplifying sizespecific radiation dose estimates in pediatric CT. AJR Am J Roentgenol 2015;204:167-76.

7. Berrington de Gonzalez A. Estimates of the potential risk of radiation-related cancer from screening in the UK. J Med Screen 2011;18:163-4.

8. Chien CR, Chen TH. Mean sojourn time and effectiveness of mortality reduction for lung cancer screening with computed tomography. Int J Cancer 2008;122:2594-9.

9. Peng $\mathrm{W}, \mathrm{Li} Z \mathrm{Z}, \mathrm{Xia} \mathrm{C}$, et al. A CONSORT-compliant prospective randomized controlled trial: radiation dose reducing in computed tomography using an additional lateral scout view combined with automatic tube current modulation: Phantom and patient study. Medicine (Baltimore) 2017;96:e7324.

10. Corwin MT, Chang M, Fananapazir G, et al. Accuracy and radiation dose reduction of a limited abdominopelvic $\mathrm{CT}$ in the diagnosis of acute appendicitis. Abdom Imaging
Li et al. Radiation doses of CT examinations and setting local DRLs

2015;40:1177-82.

11. Mathews JD, Forsythe AV, Brady Z, et al. Cancer risk in 680,000 people exposed to computed tomography scans in childhood or adolescence: data linkage study of 11 million Australians. BMJ 2013;346:f2360.

12. Hampton T. Radiation oncology organization, FDA announce radiation safety initiatives. JAMA 2010;303:1239-40.

13. Smith-Bindman R, Moghadassi M, Wilson N, et al. Radiation Doses in Consecutive CT Examinations from Five University of California Medical Centers. Radiology 2015;277:134-41.

14. Verdun FR, Gutierrez D, Vader JP, et al. CT radiation dose in children: a survey to establish age-based diagnostic reference levels in Switzerland. Eur Radiol 2008;18:1980-6.

15. RadiationprotectionN $N^{\circ} 180$ EC. Diagnostic Reference Levels in Thirty-six European Countries. Available online: https://ec.europa.eu/energy/sites/ener/files/documents/ RP180\%20part2.pdf

16. Cristy M. Mathematical Phantoms Representing Children of Various Ages for Use in Estimates of Internal Dose. Oak Ridge, Tenn: Oak Ridge National Laboratory, 1980.: U.S. Nuclear Regulatory Commission Rep. NUREG/CR1159 (also Oak Ridge National Laboratory Rep. ORNL/ NUREG/TM-367). 1980.

17. The 2007 Recommendations of the International Commission on Radiological Protection. ICRP publication 103. Ann ICRP 2007;37:1-332.

18. Medicine JAoRPi. Diagnostic Reference Levels based on latest surveys in Japan-Japan DRLs 2015. Available online: https://www.iomp.org/sites/default/files/ drlhoukokusyoeng.pdf

19. Zhou DD, Sun P, Jia Z, et al. Multisection computed tomography: Results from a Chinese survey on radiation dose metrics. J Chin Med Assoc 2019;82:155-60.

20. Zhou Y, Yang C, Cao X, et al. Survey of patients' CT radiation dose in Jiangsu Province. J Public Health Emerg 2017;1:9.

21. Suntharalingam S, Stecker FF, Guberina N, et al. How Much Is the Dose Varying between Follow-Up CTExaminations Performed on the Same Scanner with the Same Imaging Protocol? PLoS One 2016;11:e0152961.

22. Moore BM, Brady SL, Mirro AE, et al. Size-specific dose estimate (SSDE) provides a simple method to calculate organ dose for pediatric CT examinations. Med Phys 2014;41:071917.

23. Mccollough C, Bakalyar DM, Bostani M, et al. Use of 
Water Equivalent diameter for calculating patient size and size-specific dose estimates (SSDE) in CT: The report of AAPM Task Group 220.

24. Christner JA, Braun NN, Jacobsen MC, et al. Size-specific dose estimates for adult patients at CT of the torso. Radiology 2012;265:841-7.

25. Brink JA, Miller DL. U.S. National Diagnostic Reference Levels: Closing the Gap. Radiology 2015;277:3-6.

26. Goske MJ, Strauss KJ, Coombs LP, et al. Diagnostic reference ranges for pediatric abdominal CT. Radiology 2013;268:208-18.

27. Commission CNH. Diagnostic reference levels for adults in X-ray computed tomography. Available online: http://www.nhc.gov.cn/ewebeditor/uploadfi le/2018/10/20181012110201559.pdf

28. Soderberg M. Overview, practical tips and potential pitfalls

Cite this article as: $\mathrm{Li} \mathrm{Z}$, Zhang J, Xia C, Zhao F, Zhang $\mathrm{K}$, Li Y, Li L, Pu J, Peng W, Liu K, Guo Y. Radiation doses in CT examinations from the West China Hospital, Sichuan University and setting local diagnostic references levels. Ann Transl Med 2020;8(16):1010. doi: 10.21037/atm-20-5443 of using automatic exposure control in CT: siemens care dose 4D. Radiat Prot Dosimetry 2016;169:84-91.

29. Zhang J, Kang S, Han D, et al. Application of intelligent optimal $\mathrm{kV}$ scanning technology (CARE $\mathrm{kV}$ ) in dualsource computed tomography (DSCT) coronary angiography. Int J Clin Exp Med 2015;8:17644-53 .

30. Bittencourt MS, Schmidt B, Seltmann M, et al. Iterative reconstruction in image space (IRIS) in cardiac computed tomography: initial experience. Int J Cardiovasc Imaging 2011;27:1081-7.

31. De Marco P, Origgi D. New adaptive statistical iterative reconstruction $\mathrm{ASiR}-\mathrm{V}$ : Assessment of noise performance in comparison to ASiR. J Appl Clin Med Phys 2018;19:275-86.

(English Language Editor: J. Chapnic) 